\title{
Kisspeptin level in the aging ovary is regulated by the sympathetic nervous system
}

\author{
Daniela Fernandois', Gonzalo Cruz², Eun Kyung Na1, Hernán E Lara' and \\ Alfonso H Paredes ${ }^{1}$ \\ 'Department of Biochemistry and Molecular Biology, Laboratory of Neurobiochemistry, Faculty of \\ Chemistry and Pharmaceutical Sciences, Universidad de Chile, Santiago, Chile \\ 2Laboratorio de Alteraciones Reproductivas y Metabólicas, Centro de Neurobiología y Plasticidad \\ Cerebral (CNPC), Instituto de Fisiología, Facultad de Ciencias, Universidad de Valparaíso, \\ Valparaíso, Chile
}

Correspondence should be addressed to A H Paredes Email

aparedes@ciq.uchile.cl

\begin{abstract}
Previous work has demonstrated that the increase in the activity of sympathetic nerves, which occurs during the subfertility period in female rats, causes an increase in follicular cyst development and impairs follicular development. In addition, the increase in ovarian sympathetic activity of aged rats correlates with an increased expression of kisspeptin (KISS1) in the ovary. This increase in KISS1 could participate in the decrease in follicular development that occurs during the subfertility period. We aimed to determine whether the blockade of ovarian sympathetic tone prevents the increase in KISS1 expression during reproductive aging and improves follicular development. We performed 2 experiments in rats: (1) an in vivo blockade of beta-adrenergic receptor with propranolol $(5.0 \mathrm{mg} / \mathrm{kg})$ and (2) an ovarian surgical denervation to modulate the sympathetic system at these ages. We measured Kisspeptin and follicle-stimulating hormone receptor (FSHR) mRNA and protein levels by qRT-PCR and western blot and counted primordial, primary and secondary follicles at 8,10 and 12 months of age. The results showed that ovarian KISS1 decreased but FSHR increased after both propranolol administration and the surgical denervation in rats of 8 , 10 and 12 months of age. An increase in FSHR was related to an increase in the number of smaller secondary follicles and a decreased number of primordial follicles at 8, 10 and 12 months of age. These results suggest that intraovarian KISS1 is regulated by sympathetic nerves via a beta-adrenergic receptor and participates locally in ovarian follicular development in reproductive aging.
\end{abstract}

\author{
Key Words \\ - kisspeptin \\ - aging \\ - noradrenaline \\ - ovary
}

Journal of Endocrinology (2017) 232, 97-105

\section{Introduction}

Previous work has demonstrated that during the subfertility period in rats, the content of norepinephrine in the ovary increases (Chavez-Genaro et al. 2007, Acuna et al. 2009), which is accompanied by an increase in the activity of the sympathetic nerves (Acuna et al. 2009).
Owing to the consistent evidence that noradrenergic innervation of the ovary is important in ovarian physiology (Ferruz et al. 1991, Mayerhofer et al. 1997, Ricu et al. 2008) and pathophysiology in rats (Lara et al. 1993, Dorfman et al. 2003, Rosa et al. 2003, Greiner et al. 2005, 
Sotomayor-Zarate et al. 2008, Acuna et al. 2009, Fernandois et al. 2012), we believe that the increase in noradrenergic tone could affect both follicular growth and ovulation during the subfertility period. In fact, we demonstrated that the blocking of beta-adrenergic receptor with propranolol in 10- and 12-month-old rats increases the number of healthy antral follicles and corpora lutea, improves estrous cyclicity and inhibits follicular cyst formation (Fernandois et al. 2012). These results demonstrate that a chronic increase in adrenergic tone during the subfertility period may cause a decrease in follicular growth. This contradicts the previous findings that show that adrenergic stimulation in the ovary results in the increased expression of follicle-stimulating hormone receptor (FSHR), thus favoring follicular growth in neonatal rats (Mayerhofer et al. 1997). In an effort to explain this difference, we found that the increase in sympathetic activity in the ovaries of aged rats shows a correlation with an increase in Kisspeptin (KISS1) expression in the ovary (Fernandois et al. 2016). Surprisingly, in in vitro experiments, we found that KISS1 blocks the ability of isoproterenol (a beta-adrenergic agonist) to increase FSHR expression and follicular development (Fernandois et al. 2016), which may affect the acquisition of FSHR by the early-developing follicles. Previous studies published by our laboratory show that beta-adrenergic receptor activation increases the expression of Kiss 1 mRNA in rat ovaries that are incubated in vitro (Ricu et al. 2012). In the present work, using in vivo approaches, we aimed to demonstrate that either propranolol administration or surgical denervation of the ovary may possibly decrease intraovarian KISS1 and, consequently, increase follicular growth.

\section{Materials and methods}

\section{Animals}

Sprague-Dawley rats from the animal facility of the Faculty of Chemistry and Pharmaceutical Science of the University of Chile were used. All rats remained in a temperature controlled room, with a 12-h light and 12-h darkness cycle (the light cycle began at $0700 \mathrm{~h}$ ) and access to food and water ad libitum. The Bioethics Committee of the Faculty of Chemistry and Pharmaceutical Science of Universidad de Chile and the Institutional Animal Experimentation Bioethics Board and the Science Council (FONDECYT) of Chile approved all experimental procedures (code CBE2013-15). All efforts were taken to minimize the number of animals used, and all procedures were undertaken in a humane manner.
For the experiment with propranolol, rats of 4 months (saline $n=5$ ), 6 months (saline $n=5$, propranolol $n=5$ ), 8 months (saline $n=5$, propranolol $n=9$ ) and 10 months of age (saline $n=5$, propranolol $n=7$ ) were used. DL-propranolol $\mathrm{HCl} 5 \mathrm{mg} / \mathrm{kg}$ of weight (SigmaAldrich) was freshly prepared and administered daily by an intraperitoneal injection for two months, whereas controls received only vehicle (saline solution). Injections were given for two months so the drug would affect at least one complete cohort of follicles (McGee \& Hsueh 2000). At the end of the two months, all rats were killed by decapitation, and their tissues were collected and stored at $-80^{\circ} \mathrm{C}$ until analysis. To dissect the effect of the sympathetic system over the ovary, we performed a second experiment, in which a physical denervation of the ovarian superior nerve was done according to the previously reported protocol (Barria et al. 1993, Rosa et al. 2003). In brief, both ovaries were exposed and a bilateral cut of the nerve was performed. Sham animals were subjected to the same surgery, in which ovaries were exposed and manipulated but without cutting the nerve. Rats aged 5 months (sham $n=5$ ), 7 months (sham $n=5$, SONX $n=5$ ), 9 months ( and 11 months (sham $n=10$, SONX $n=6$ ) were used. Euthanasia and tissue collection were performed 20 days after the denervation procedure.

\section{Real-time PCR}

The total RNA was extracted using an E.Z.N.A. Total RNA kit (Omega-Biotek, Norcross, GA, USA). In total, $2 \mu \mathrm{g}$ of total RNA was subjected to reverse transcription. For real-time PCR, we used Brilliant II SYBR Green QPCR Master Mix (Agilent Technologies) in an IQ5 thermocycler (Bio-Rad Laboratories). Primer-specific amplification and quantification cycles were run at $95^{\circ} \mathrm{C}$ for $20 \mathrm{~s}, 62.5^{\circ} \mathrm{C}$ for $20 \mathrm{~s}$ for Kiss $1\left(60^{\circ} \mathrm{C}\right.$ for Fshr and $\left.18 \mathrm{~s}\right)$, $72^{\circ} \mathrm{C}$ for $20 \mathrm{~s}$ and a final extension of $72^{\circ} \mathrm{C}$ for $10 \mathrm{~min}$. To normalize the quantification of Kiss 1 and Fshr mRNA, we measured the amount of ribosomal 18s RNA in each protocol using a commercially available RT primer pair (Ambion). The corresponding standard curve for each gene was obtained by the serial dilution of known copy numbers of the purified amplification PCR product of each gene using Diffinity RapidTips (Diffinity Genomics, Inc.). The primers used for each product were used in previous studies (Fernandois et al. 2016) and included the following: Fshr: sense 5'-CAT CAC TGT GTC CAA GGC CA-3' and antisense 5'-TGC GGA AGT TCT TGG TGA AAA-3', GenBank accession number AF095642; and

Published by Bioscientifica Ltd. 
for Kiss1: sense 5'-CCG GAC CCC AGG AAC TCG T-3' and antisense 5'-CG TAG CGC AGG CCA AAG GAG-3', GenBank accession number NM_181692.

\section{Western blot}

The detection of ovarian KISS1 and the internal control GAPDH in all experiments was performed by western blot, following separation by SDS-PAGE on $15 \%$ polyacrylamide gels under reducing conditions. The proteins were transferred to nitrocellulose membranes, blocked with $5 \%$ milk for $1 \mathrm{~h}$ and probed with rabbit polyclonal antiKISS1 antibody (ab19028, Abcam) in a 1:50 dilution overnight, a 1:40,000 dilution of rabbit polyclonal antiGAPDH (antibody G9545, Sigma-Aldrich) or in a 1:10,000 dilution of rabbit polyclonal anti-FSH receptor (antibody NBP1-4630, Novus Biologicals, Littleton, CO, USA) for $1 \mathrm{~h}$. The antibody complexes were detected using goat anti-rabbit IgG Fc (HRP) (ab97200, Abcam) at 1:10,000, and for chemiluminescence, an EZ-ECL Enhanced Chemiluminescence Detection Kit (Biological Industries,
$\mathrm{KBH}$, Israel) was used. Chemiluminescence was captured using a G-Box Syngene system (Syngene Headquarters, MD, USA).

\section{Plasma FSH evaluation}

Plasma FSH was assessed using a rat follicle-stimulating hormone ELISA Kit (CSB-E06869r, Cusabio Biotech, Wuhan, China) according to the manufacturer's instructions. The ELISA detection range was $0.13-10 \mathrm{IU} / \mathrm{mL}$, and the sensitivity limit was $0.07 \mathrm{IU} / \mathrm{mL}$. No significant cross-reactivity or interference between rat FSH and analogues was detected according to the manufacturer's information.

\section{Histological analysis}

Ovaries were immersed in Bouin's fixative immediately after killing. They were then embedded in paraffin, cut into $6-\mu \mathrm{m}$ sections and stained with hematoxylin and eosin. The counting of primordial, primary and secondary
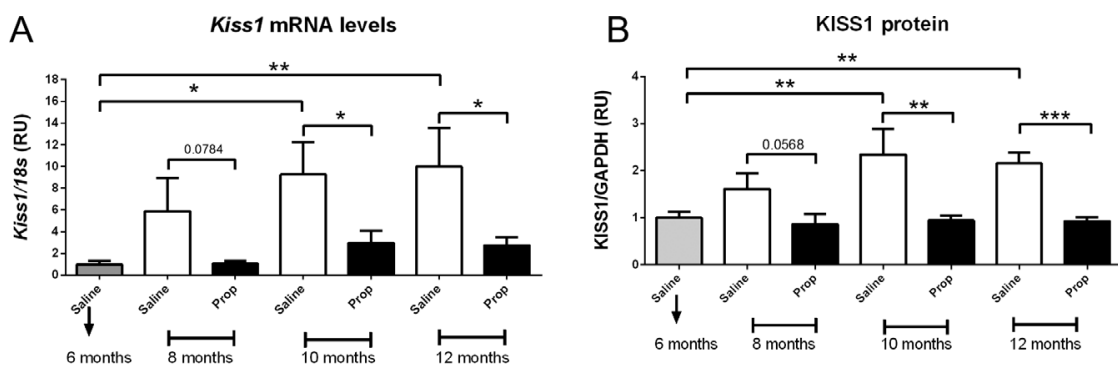

C
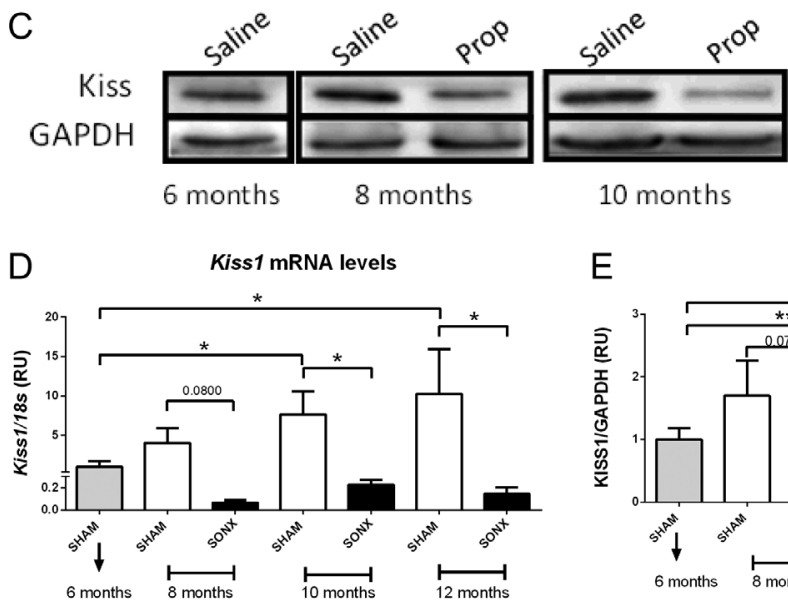

10 months

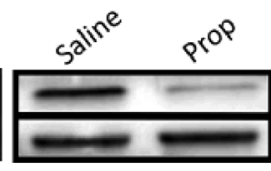

12 months

E

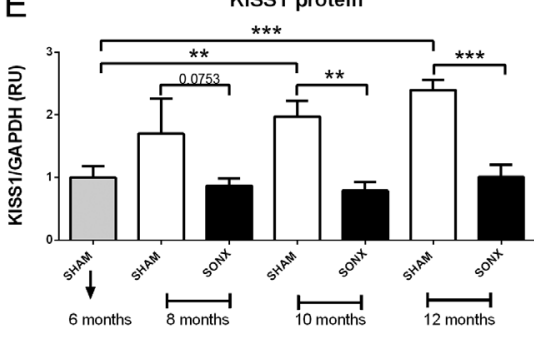

F

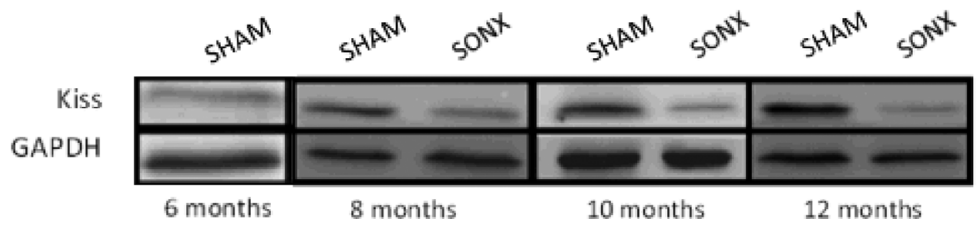

\section{Figure 1}

Kisspeptin expression decreases with a

$\beta$-adrenergic blockade or a surgical denervation (SONX). A, B, C indicate changes in propranololtreated rats and $D, E, F$ indicate SONX experiments. (A) and (D) indicate Kiss1 transcript levels in the ovaries of Sprague-Dawley rats quantified by RT-qPCR. The mRNA levels were normalized to the $18 \mathrm{~s}$. (B) and (E) protein levels quantified by western blot; each sample was assessed at least 3 times. Kisspeptin values were normalized to GAPDH. Pixels were counted using the ImageJ software. (C) and (F) are representative images of the quantification of (B) and $(E)$, respectively. Significance was obtained using Student's $t$-test. ${ }^{*} P<0.05, * * P<0.01$ and $* * * P<0.001$. Differences between conditions are indicated above each bar. The results are expressed as the means \pm s.E.M. For mRNA levels: 6 months: saline $N=5$ and sham $N=5 ; 8$ months: saline $N=5$, propranolol $N=7$, sham $N=5$ and SONX $=5 ; 10$ months: saline $=5$ and propranolol $N=9$, sham $N=5$ and SONX $N=5 ; 12$ months: saline $N=5$, propranolol $N=7$, Sham $N=6$ and SONX $N=5$. For western blot, $N=5$ for saline, propranolol, sham and SONX groups of every age. 
A

Fshr mRNA

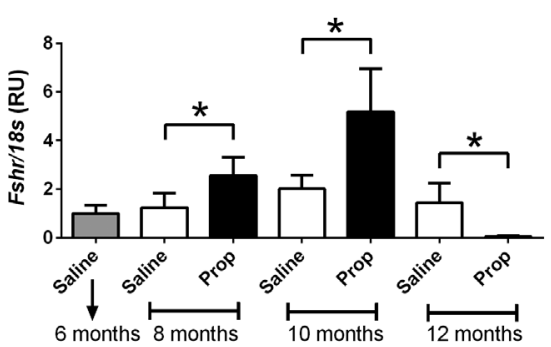

C
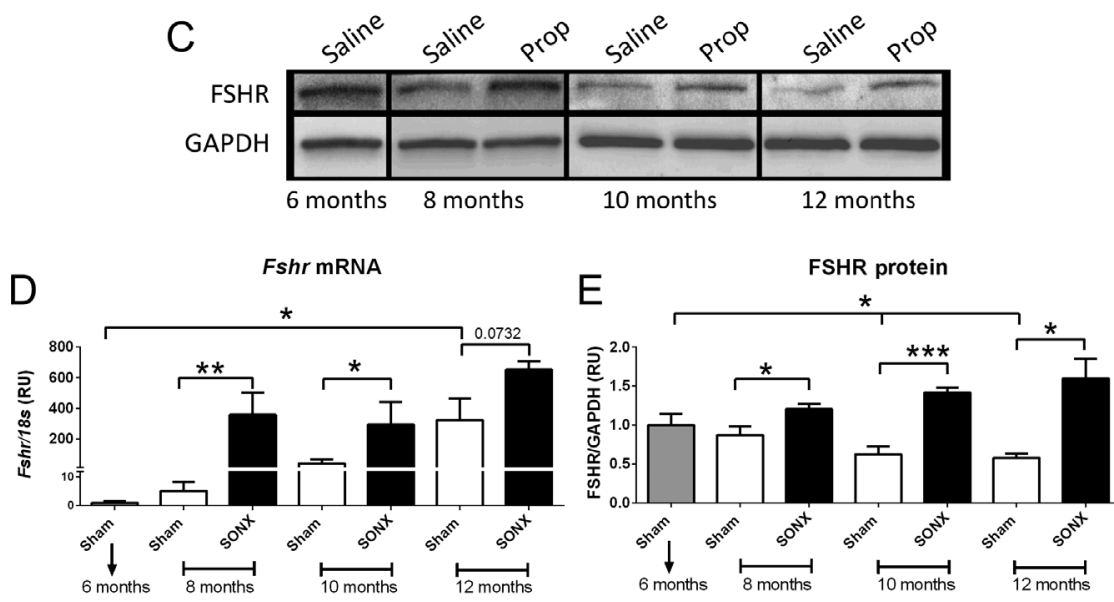

F

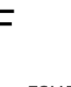

FSHR

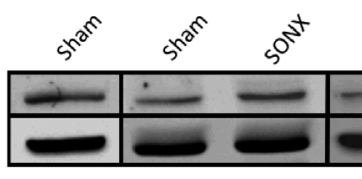

6 months
B

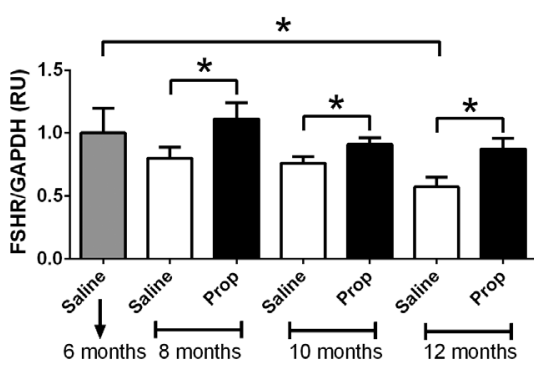

12 months
Figure 2

FSHR expression is increased in the ovaries. A, B, $C$ indicate changes in propranolol-treated rats and $D, E, F$ indicate SONX experiments. (A) and (D) indicate Fshr transcript levels in the ovaries of Sprague-Dawley rats. The mRNA was quantified by RT-qPCR. mRNA levels were normalized to the 18s. (B) and (E) indicate protein levels obtained by western blot; each sample was assayed at least 3 times. FSHR values were normalized to GAPDH. Pixels were counted using the ImageJ software. (C) and (F) are representative images of the quantification of (B) and (E), respectively. Significance was determined using Student's $t$-test. ${ }^{*} P<0.05, * * P<0.01$ and $* * * P<0.001$. Differences between conditions are indicated above each bar. The results are expressed as the means \pm S.E.M. For mRNA levels: 6 months: saline $N=5$ and sham $N=5 ; 8$ months: saline $N=5$, propranolol $N=7$, Sham $N=5$ and SONX $=5$; 10 months: saline $=5$ and propranolol $N=9$, sham $N=5$ and SONX $N=5 ; 12$ months: saline $N=5$, propranolol $N=7$, sham $N=6$ and SONX $N=5$. For western blot, $N=4$ for saline, propranolol, sham and SONX groups of every age. follicles was performed according to previous reports (Lara et al. 2000, Cruz et al. 2012). All consecutive slices were observed and analyzed for primary and secondary follicles counting. Both primary and secondary follicles were scored when the nucleolus of the oocyte was visible (usually when the diameter of the follicle is the largest). Therefore, the total number of primary and secondary follicles was counted in each ovary. On the other hand, to avoid overcounting, primordial follicles were counted each 3 slices if the nucleus of the oocyte was visible. The follicles were classified according to their histological characteristics. In brief, primordial follicles were scored if they contained an intact oocyte with a visible nucleus surrounded by a single layer of flattened granulosa cells.
Follicles were scored as primary if they consisted of an intact, enlarged oocyte with a visible nucleolus and a single layer of cuboidal granulosa cells. Follicles were scored as secondary if they contained an oocyte with a visible nucleolus and two or more layers of granulosa cells but without antral cavity.

\section{Statistical analysis}

Graphs were plotted as the fold of change over the mean value for the 6-month-old rats. The group of 6-month-old rats was used as a control of young rats. The differences between age groups were assessed using an ANOVA, followed by Fisher's test. The differences between saline

Table 1 Serum FSH levels in propranolol-treated rats and SONX rats. ${ }^{*} P<0.05$.

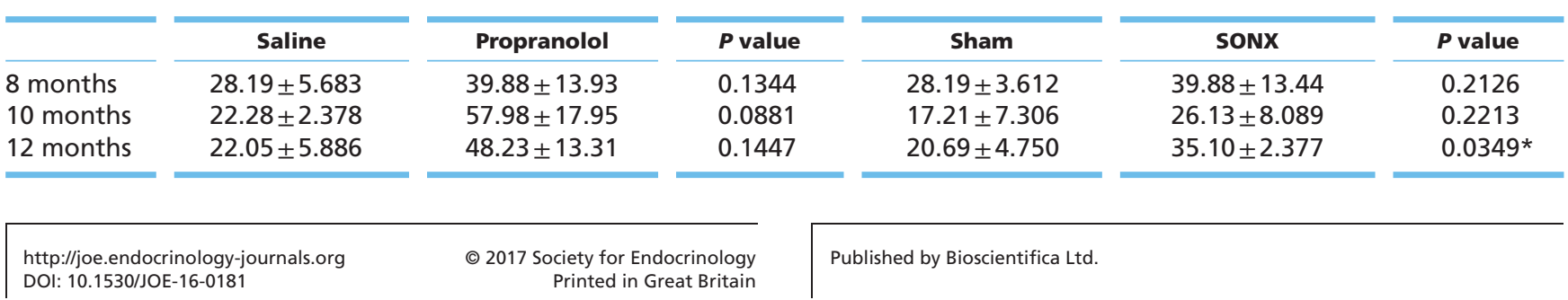


A

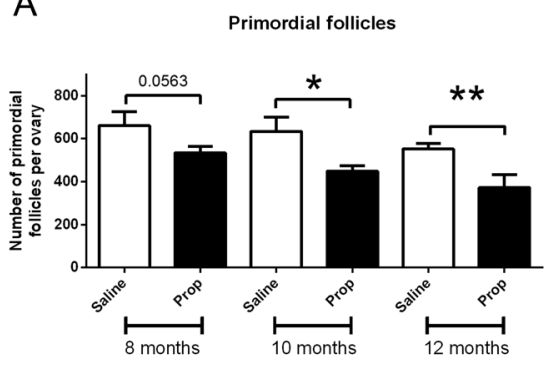

B

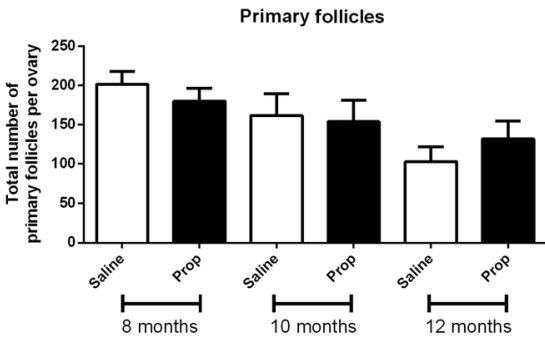

C

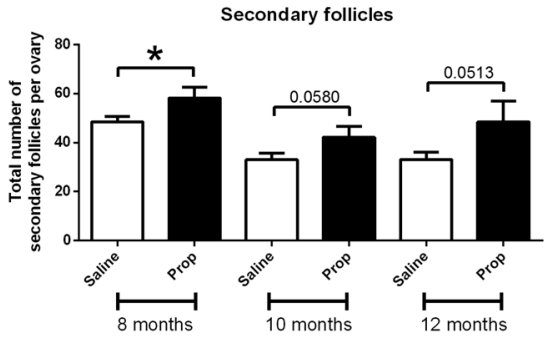

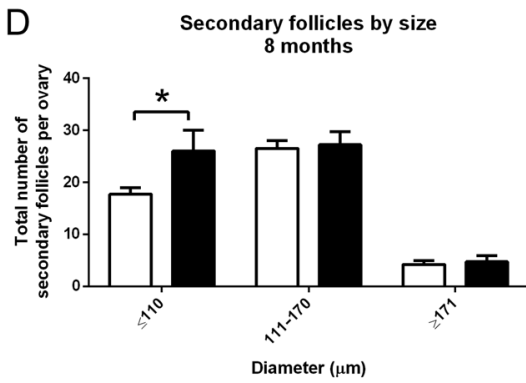

E Secondary follicles by size

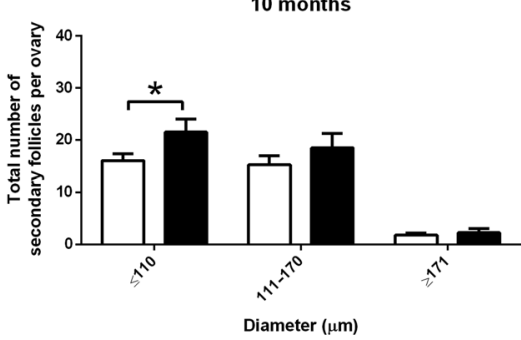

$\mathrm{F}$

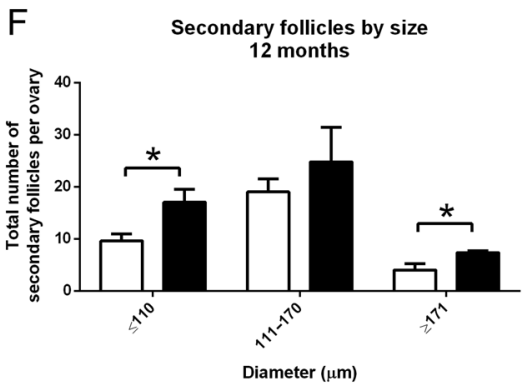

Figure 3

Total number of preantral follicles in subfertile ovaries with a beta-adrenergic pharmacological blockade. Total number of follicles counted in 4 ovaries for controls and 5 ovaries for propranolol condition. (A) Number of primordial follicles per ovary (counted every 3 slices), (B) total number of primary follicles per ovary, $(C)$ total number of secondary follicles per ovary. (D, E, F) Total number of secondary follicles per ovary grouped by size at $8(D), 10(E)$ and 12 months of age (F). Significance was determined using Student's $t$-test between the saline and propranolol group of the same age. ${ }^{*} P<0.05$ and $* * P<0.01$. Asterisks represent differences against saline (control) ovaries. The results are expressed as the means \pm s.E.M. and propranolol or sham and SONX for the same age group were assessed using an unpaired Student's t-test. $P<0.05$ was considered to be statistically significant. The statistical analyses were performed using GraphPad Prism software, version 6.0 (GraphPad Software).

\section{Results}

\section{Propranolol administration and surgical denervation during the subfertility period in the rat decreases KISS1 expression in the ovary}

To observe the effect of beta-adrenergic blockade on ovarian KISS1 expression, we administered propranolol for two months, starting with 3 groups of rats that were 6, 8 and 10 months old. At the end of the treatment, we measured the mRNA and protein Kisspeptin levels. At 10 and 12 months of age, propranolol decreased the mRNA levels and the peptide KISS1 compared with the control (saline) (Fig. 1A and B). Because the propranolol was administered intraperitoneally, it could affect both the central and peripheral action of the sympathetic system.
To selectively reduce the adrenergic tone of the ovary, we performed a surgical cut of ovarian superior nerve (SONX). Twenty days after denervation, a decrease in the mRNA levels and the protein KISS1 was observed (Fig. 1D and E). No significant changes in the kisspeptin mRNA and protein were observed at 8 months of age in either experiment, but a tendency to decrease was observed in the propranolol group $(P=0.0784$ for mRNA and $P=0.0568$ for protein) and in the SONX experiment $(P=0.0800$ for mRNA and $P=0.0753$ for protein). Importantly, we observed an increase in KISS1 expression in the 10and 12-month-old saline and sham rats compared with 6-month-old saline and sham rats, respectively (Fig. 1). This result is consistent with our previous publication (Fernandois et al. 2016).

\section{Propranolol administration and surgical denervation during the subfertility period in the rat increases FSHR expression in the ovary}

We have previously shown that KISS1 can regulate the growth of follicles by modulating the expression 

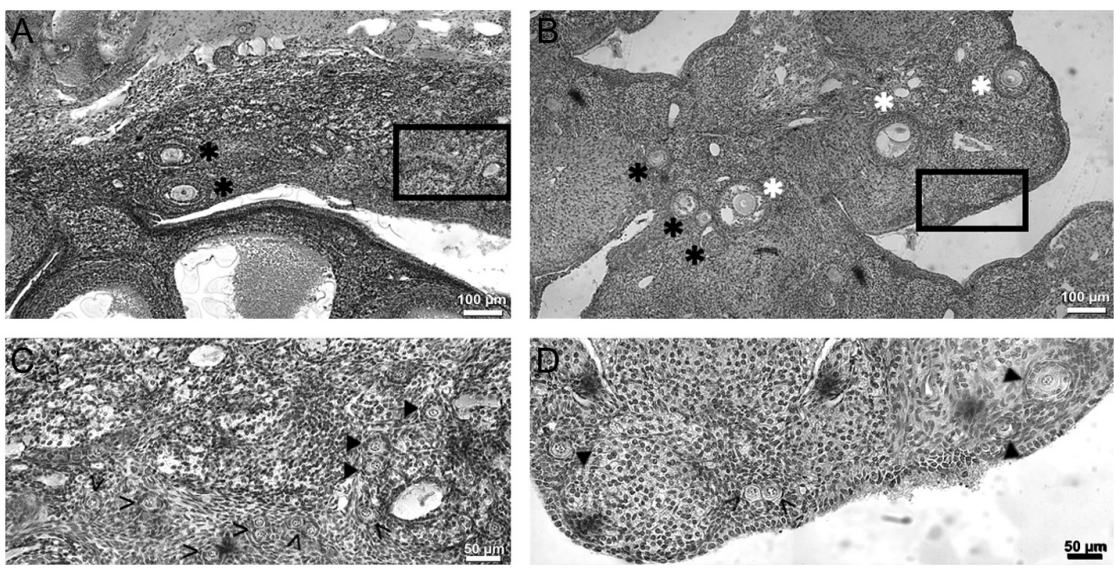

\section{Figure 4}

Representative pictures of ovarian histology from rats treated with propranolol for two months. The figure shows representative pictures of ovaries treated with saline solution (A: 100x, C: $400 x$ ) or propranolol $5 \mathrm{mg} / \mathrm{kg}$ i.p. from 8 to 10 months of age (B: 100x, D: $400 x$ ). In both (A) and (B), black asterisk indicates secondary follicles, and white asterisk indicates antral follicles. In both (C) and (D), open arrows indicate primordial follicles and black filled arrows primary follicles. of FSHR (Fernandois et al. 2016). Because propranolol administration and surgical denervation of the SON decrease the KISS1 levels in the ovary, we measured FSHR to observe the follicular response. Propranolol produced a significant increase in Fshr mRNA levels in 8- and 10-month-old rats. However, propranolol unexpectedly produced a decrease in Fshr in 12-month-old rats, as shown in Fig. 2A. In addition, the surgical denervation of the ovary increased the mRNA levels of Fshr in rats that were 8,10 and 12 months of age (Fig. 2B). In the same way, the FSHR protein levels increased in both experimental groups (pharmacological blockade and surgical denervation) at 8,10 and 12 months of age compared with their own controls (Fig. 2B, C, E and F). In contrast, the plasma FSH levels did not change with propranolol treatment in all ages. In addition, only the SONX group at 12 months of age showed an increased FSH level (Table 1).

\section{Propranolol administration within the subfertility period in the rat improves the transition of primordial to secondary follicles in the ovary}

Because we hypothesized that increases in FSHR should favor early follicular development, we quantified the primordial, primary and secondary follicles in the propranolol group. In Fig. $3 \mathrm{~A}$, we show that after propranolol administration, the number of primordial follicles tended to decrease in 8-month-old rats $(P=0.0563)$, whereas the number of secondary follicles increased. In addition, the number of primordial follicles decreased in 10- and 12-month-old rats, whereas the number of secondary follicles tended to increase $(P=0.0580$ and $P=0.0513$ for 10 - and 12 -month-old rats, respectively, Fig. 3C). No significant changes were observed in primary follicles (Fig. 3B). We also found that the number of the secondary follicles $\leq 110 \mu \mathrm{m}$ was increased at all ages (Fig. 3D, E and F). Figure 4 illustrates a representative image in which some clusters of primordial follicles are found in the saline group (Fig. 4C, open arrows), but this is more difficult to find in the propranolol group because primordial follicles are more scattered (Fig. 4D, open arrows). In the same line, more developing follicles were found in the propranolol group than those in the saline group (Fig. 4A and B).

\section{Discussion}

We have recently demonstrated that KISS1 expression in the ovary increases with age during the subfertility period in rats (Fernandois et al. 2016). In addition, we found a correlation between norepinephrine release and the levels of KISS1 during this period. In the present work, we found that either blocking beta-adrenergic receptors in the ovary or sectioning the SON produces a decrease in KISS1 levels, whichisfunctionally related totheincreasein FSHR and favors follicular development. This supports the idea that KISS1 is regulated by the sympathetic nervous system and that both systems are complementary in the regulation of follicular development.

Kisspeptin has been the subject of intense research due to its essential role in the regulation of the gonadotropic axis at the central nervous system level, including the regulation of puberty (Castellano et al. 2009, Roa et al. 2009, Pineda et al. 2010, Pinilla et al. 2012, Tena-Sempere 2013). Despite this fundamental role in the central nervous system, KISS1 is expressed in different tissues, including the ovary (Castellano et al. 2006, Bhattacharya \& Babwah 2015, Fayazi et al. 2015, Wahab et al. 2016). Interestingly, ovarian KISS1 has been associated with ovulation due to an increase in Kiss1 mRNA levels during proestrus (Castellano et al. 2006, Laoharatchatathanin et al. 2015).

Published by Bioscientifica Ltd 
In addition, blocking ovulation with indomethacin prevents this increase in ovarian Kiss1 mRNA levels (Gaytan et al. 2009). Furthermore, we recently found that KISS1 locally infused in the ovary with a miniosmotic pump increases the number of corpora lutea in 6- and 10-month-old rats, thus reinforcing the idea that KISS1 favors ovulation (Fernandois et al. 2016). However, we also found a decrease in the number and size of antral follicles, which suggests that KISS1 delays follicular development.

In the present work, we observed a decrease in Kisspeptin mRNA and protein by blocking betaadrenergic receptors with propranolol i.p. for 2 months. This decrease in KISS1 is more significant in 10- and 12-month-old rats, probably because at these ages, the increase in the noradrenergic tone of the ovary is more evident (Acuna et al. 2009). Because propranolol could produce the effect at different levels (e.g., hypothalamus, celiac ganglion), we aimed to isolate the specific ovarian noradrenergic effect by surgically cutting the SON. As seen with the pharmacological blockade with propranolol, SON denervation was more effective in reducing Kisspeptin mRNA and protein in 10- and 12-month-old rats. Both results (using propranolol administration and SON denervation) are in concordance with the fact that in vitro, the incubation of ovaries with isoproterenol (a beta-adrenergic agonist) increases KISS1 expression (Ricu et al. 2012). A possible molecular explanation involves the cAMP/PKA signaling pathway. Beta-adrenergic receptors are associated with the Gs protein, and its activation results in the increased production of cAMP. Then, cAMP activates the cAMP-dependent protein kinase (PKA), which translocates into the nucleus and activates the transcription factor CREB (Montminy 1997). The Kiss1 promoter has an upstream region that contains a Cre sequence, which allows the binding of CREB (Altarejos et al. 2008). A previous study demonstrated a direct regulation of KISS1 expression via the Cre region in the hypothalamus. The same regulation for Kiss1 expression was found in the liver (Song et al. 2014). Futures studies are needed to determine whether the mentioned mechanisms are responsible for the regulation of norepinephrine on Kiss1 expression in the ovary. Additionally, it will be important to confirm whether the decreased KISS1 expression after in vivo propranolol treatment is carried out in the same cells that respond to the beta-adrenergic blockade by immunohistochemistry analysis.

Regarding the physiological role of intraovarian KISS1 during the subfertility period, we previously demonstrated that KISS1 prevents the increase in both FSHR expression and follicular growth induced by an in vitro betaadrenergic stimulation with isoproterenol in aging rats (Fernandois et al. 2016). In accordance with these results, in the present work, we found that the decrease in KISS1 produced by lowering the adrenergic stimulation of the ovary in vivo resulted in higher levels of Fshr mRNA in 8- and 10-month-old rats, whereas FSHR protein was consistently increased in 8-, 10- and 12-month-old rats under the treatment with propranolol. The inconsistency between mRNA (decreases) and protein (increases) in the 12-month-old propranolol-treated rats may be due to an increased translational rate from Fshr mRNA into FSHR protein; however, we do not have evidence supporting this suggestion. Nonetheless, we strongly believe that the increase in FSHR protein coherently explains the increase in both the number of antral follicles observed (Fernandois et al. 2012) and the development of preantral follicles observed in this work and discussed below.

Interestingly, the decreased number of primordial follicles observed in the rats treated with propranolol (Fig. 3A) could reflect an increased primordial follicle activation and transition into developing follicles. In this sense, although we did not observe changes in the number of primary follicles, we can see a tendency of the secondary follicles to increase at 10 and 12 months of age $(P=0.0580$ for 10 months and $P=0.0513$ for 12 months) and an increase in the number of secondary follicles at 8 months of age. Furthermore, the significant increase in secondary follicles $\leq 110 \mu \mathrm{m}$ at all ages indicates that the transition rate from primary to secondary follicles is increased. Because FSHR is not expressed in primordial follicles, the increase in the recruitment of primordial follicles appears to be an indirect consequence of the increased rate of growth of developing follicles more than a direct effect of KISS1 on primordial activation. However, because Kiss1R is also expressed in oocytes, the decrease in KISS1 may directly affect primordial follicle activation. This possibility is interesting because the haplo-insufficient Kiss1r mice have a precocious ovarian aging due to a loss of oocytes. Although some authors (Gaytan et al. 2014) attribute this loss of oocytes to an increase in its death rate, this was indirectly estimated in oocytes of developing follicles in 32-week-old mice, and the possibility of an increase in the activation of primordial follicle should not be excluded.

The results of the present work provide evidence supporting the fact that local ovarian KISS1 could be involved in the decrease in the follicular count observed during the subfertility period and that this mechanism 
would be controlled by an increase in the activity of sympathetic nerves of the ovary that also increase during this period (Acuna et al. 2009, Fernandois et al. 2012, 2016). Although the pharmacologic effect of propranolol in other tissues (i.e., central nervous system) cannot be excluded, the absence of changes in the FSH serum levels at all ages endorses the local ovarian effect more than an effect on the hypothalamus-pituitary axis. Taken together, our findings suggest that blocking the sympathetic stimulation of the ovary favors the recruitment and growth of early-developing follicles during the subfertility period. This involves the functional antagonism of KISS1 with norepinephrine on the expression of FSHR. These results, along with previous findings (Fernandois et al. 2012 , 2016), suggest the possibility of intervening and modulating follicular development by controlling the sympathetic activity during the subfertility period.

\section{Declaration of interest}

The authors declare that there is no conflict of interest that could be perceived as prejudicing the impartiality of the research reported.

\section{Funding}

This work was supported by a Fondecyt grant (number 1120147, to Alfonso Paredes) and a Conicyt grant for doctoral thesis (number 21120454, to Daniela Fernandois).

\section{Author contribution statement}

D F determined Kiss1, Fshr, 18s and by real-time PCR; determined KISS1 and GAPDH by Western blot; participated in the ovarian morphology analysis. E K N participated in the ovarian morphology analysis; $\mathrm{H} \mathrm{L}$ : participated in the experiment of surgical superior ovarian nerve transection; G C contributed to discussion, analysis of the results and manuscript review and A P contributed to the development, design, and coordination of the research, manuscript preparation and responsibility for the grant. All of the authors read and approved the final manuscript.

\section{References}

Acuna E, Fornes R, Fernandois D, Garrido MP, Greiner M, Lara HE \& Paredes AH 2009 Increases in norepinephrine release and ovarian cyst formation during ageing in the rat. Reproductive Biology and Endocrinology 7 64. (doi:10.1186/1477-7827-7-64)

Altarejos JY, Goebel N, Conkright MD, Inoue H, Xie J, Arias CM, Sawchenko PE \& Montminy M 2008 The Creb1 coactivator Crtc1 is required for energy balance and fertility. Nature Medicine $\mathbf{1 4}$ 1112-1117. (doi:10.1038/nm.1866)

Barria A, Leyton V, Ojeda SR \& Lara HE 1993 Ovarian steroidal response to gonadotropins and beta-adrenergic stimulation is enhanced in polycystic ovary syndrome: role of sympathetic innervation. Endocrinology 133 2696-2703. (doi:10.1210/endo.133.6.8243293)
Bhattacharya M \& Babwah AV 2015 Kisspeptin: beyond the brain. Endocrinology 156 1218-1227. (doi:10.1210/en.2014-1915)

Castellano JM, Gaytan M, Roa J, Vigo E, Navarro VM, Bellido C, Dieguez C, Aguilar E, Sanchez-Criado JE, Pellicer A, et al. 2006 Expression of KiSS-1 in rat ovary: putative local regulator of ovulation? Endocrinology 147 4852-4862. (doi:10.1210/en.2006-0117)

Castellano JM, Roa J, Luque RM, Dieguez C, Aguilar E, Pinilla L \& Tena-Sempere M 2009 KiSS-1/kisspeptins and the metabolic control of reproduction: physiologic roles and putative physiopathological implications. Peptides 30 139-145. (doi:10.1016/j. peptides.2008.06.007)

Cruz G, Barra R, Gonzalez D, Sotomayor-Zarate R \& Lara HE 2012 Temporal window in which exposure to estradiol permanently modifies ovarian function causing polycystic ovary morphology in rats. Fertility and Sterility 98 1283-1290. (doi:10.1016/j. fertnstert.2012.07.1060)

Chavez-Genaro R, Lombide P, Dominguez R, Rosas P \& Vazquez-Cuevas F 2007 Sympathetic pharmacological denervation in ageing rats: effects on ovulatory response and follicular population. Reproduction, Fertility and Development 19 954-960. (doi:10.1071/RD07075)

Dorfman M, Arancibia S, Fiedler JL \& Lara HE 2003 Chronic intermittent cold stress activates ovarian sympathetic nerves and modifies ovarian follicular development in the rat. Biology of Reproduction $\mathbf{6 8}$ 2038-2043. (doi:10.1095/biolreprod.102.008318)

Fayazi M, Calder M, Bhattacharya M, Vilos GA, Power S \& Babwah AV 2015 The pregnant mouse uterus exhibits a functional kisspeptin/ KISS1R signaling system on the day of embryo implantation. Reproductive Biology and Endocrinology 13 105. (doi:10.1186/s12958015-0105-1)

Fernandois D, Lara HE \& Paredes AH 2012 Blocking of beta-adrenergic receptors during the subfertile period inhibits spontaneous ovarian cyst formation in rats. Hormone and Metabolic Research 44 682-687. (doi:10.1055/s-0032-1304607)

Fernandois D, Na E, Cuevas F, Cruz G, Lara HE \& Paredes AH 2016 Kisspeptin is involved in ovarian follicular development during aging in rats. Journal of Endocrinology 228 161-170. (doi:10.1530/JOE-150429)

Ferruz J, Barria A, Galleguillos X \& Lara HE 1991 Release of norepinephrine from the rat ovary: local modulation of gonadotropins. Biology of Reproduction 45 592-597. (doi:10.1095/ biolreprod45.4.592)

Gaytan F, Gaytan M, Castellano JM, Romero M, Roa J, Aparicio B, Garrido N, Sanchez-Criado JE, Millar RP, Pellicer A, et al. 2009 KiSS-1 in the mammalian ovary: distribution of kisspeptin in human and marmoset and alterations in KiSS-1 mRNA levels in a rat model of ovulatory dysfunction. American Journal of Physiology: Endocrinology and Metabolism 296 E520-E531. (doi:10.1152/ajpendo.90895.2008)

Gaytan F, Garcia-Galiano D, Dorfman MD, Manfredi-Lozano M, Castellano JM, Dissen GA, Ojeda SR \& Tena-Sempere M 2014 Kisspeptin receptor haplo-insufficiency causes premature ovarian failure despite preserved gonadotropin secretion. Endocrinology $\mathbf{1 5 5}$ 3088-3097. (doi:10.1210/en.2014-1110)

Greiner M, Paredes A, Araya V \& Lara HE 2005 Role of stress and sympathetic innervation in the development of polycystic ovary syndrome. Endocrine 28 319-324. (doi:10.1385/ENDO:28:3:319)

Laoharatchatathanin T, Terashima R, Yonezawa T, Kurusu S \& Kawaminami M 2015 Augmentation of metastin/kisspeptin mRNA expression by the proestrous luteinizing hormone surge in granulosa cells of rats: implications for luteinization. Biology of Reproduction 93 15. (doi:10.1095/biolreprod.115.127902)

Lara HE, Ferruz JL, Luza S, Bustamante DA, Borges Y \& Ojeda SR 1993 Activation of ovarian sympathetic nerves in polycystic ovary syndrome. Endocrinology 133 2690-2695. (doi:10.1210/endo.133.6.7902268)

Lara HE, Dissen GA, Leyton V, Paredes A, Fuenzalida H, Fiedler JL \& Ojeda SR 2000 An increased intraovarian synthesis of nerve growth factor and its low affinity receptor is a principal component of 
steroid-induced polycystic ovary in the rat. Endocrinology 141 1059-1072. (doi:10.1210/endo.141.3.7395)

Mayerhofer A, Dissen GA, Costa ME \& Ojeda SR 1997 A role for neurotransmitters in early follicular development: induction of functional follicle-stimulating hormone receptors in newly formed follicles of the rat ovary. Endocrinology 138 3320-3329. (doi:10.1210/ endo.138.8.5335)

McGee EA \& Hsueh AJ 2000 Initial and cyclic recruitment of ovarian follicles. Endocrine Reviews 21 200-214. (doi:10.1210/edrv.21.2.0394)

Montminy M 1997 Transcriptional regulation by cyclic AMP. Annual Review of Biochemistry 66 807-822. (doi:10.1146/annurev. biochem.66.1.807)

Pineda R, Garcia-Galiano D, Roseweir A, Romero M, Sanchez-Garrido MA, Ruiz-Pino F, Morgan K, Pinilla L, Millar RP \& Tena-Sempere M 2010 Critical roles of kisspeptins in female puberty and preovulatory gonadotropin surges as revealed by a novel antagonist. Endocrinology 151 722-730. (doi:10.1210/en.2009-0803)

Pinilla L, Aguilar E, Dieguez C, Millar RP \& Tena-Sempere M 2012 Kisspeptins and reproduction: physiological roles and regulatory mechanisms. Physiological Reviews 92 1235-1316. (doi:10.1152/ physrev.00037.2010)

Ricu M, Paredes A, Greiner M, Ojeda SR \& Lara HE 2008 Functional development of the ovarian noradrenergic innervation. Endocrinology 149 50-56. (doi:10.1210/en.2007-1204)

Ricu MA, Ramirez VD, Paredes AH \& Lara HE 2012 Evidence for a celiac ganglion-ovarian kisspeptin neural network in the rat: intraovarian anti-kisspeptin delays vaginal opening and alters estrous cyclicity. Endocrinology 153 4966-4977. (doi:10.1210/ en.2012-1279)

Roa J, Castellano JM, Navarro VM, Handelsman DJ, Pinilla L \& TenaSempere M 2009 Kisspeptins and the control of gonadotropin secretion in male and female rodents. Peptides 30 57-66. (doi:10.1016/j.peptides.2008.08.009)

Rosa ESA, Guimaraes MA, Padmanabhan V \& Lara HE 2003 Prepubertal administration of estradiol valerate disrupts cyclicity and leads to cystic ovarian morphology during adult life in the rat: role of sympathetic innervation. Endocrinology 144 4289-4297. (doi:10.1210/ en.2003-0146)

Song WJ, Mondal P, Wolfe A, Alonso LC, Stamateris R, Ong BW, Lim OC, Yang KS, Radovick S, Novaira HJ, et al. 2014 Glucagon regulates hepatic kisspeptin to impair insulin secretion. Cell Metabolism 19 667-681. (doi:10.1016/j.cmet.2014.03.005)

Sotomayor-Zarate R, Dorfman M, Paredes A \& Lara HE 2008 Neonatal exposure to estradiol valerate programs ovarian sympathetic innervation and follicular development in the adult rat. Biology of Reproduction 78 673-680. (doi:10.1095/biolreprod.107.063974)

Tena-Sempere M 2013 Keeping puberty on time: novel signals and mechanisms involved. Current Topics in Developmental Biology 105 299-329. (doi:10.1016/b978-0-12-396968-2.00011-7)

Wahab F, Atika B, Shahab M \& Behr R 2016 Kisspeptin signalling in the physiology and pathophysiology of the urogenital system. Nature Reviews Urology 13 21-32. (doi:10.1038/nrurol.2015.277)

Received in final form 12 October 2016

Accepted 17 October 2016
๑) 2017 Society for Endocrinology Printed in Great Britain
Published by Bioscientifica Ltd 